\title{
THE STATIONARY STATES OF INTERACTING FIELDS
}

\author{
by W. R. FRAZER *) and L. VAN HOVE \\ Instituut voor theoretische fysica der Rijksuniversiteit, Utrecht, Nederland
}

\section{Synopsis}

As an application of a time-independent perturbation formalism developed earlier for systems with many degrees of freedom, we give in terms of diagrams the general perturbation expressions for the exact stationary states of interacting fields. The physical vacuum is obtained by applying to the bare vacuum the exponential of an operator simply expressed in terms of connected diagrams. For the states with one or more physical particles expressions are found in the form of suitably defined creation operators applied to the physical vacuum. The incoming and outgoing parts of a scattering state are analyzed in terms of products of physical particle creation operators, the connection being given by the S-matrix. Some remarks are made on renormalization. For electrodynamics in particular the ratio of renormalized to unrenormalized fine structure constants is shown to have a simple probabilistic interpretation: it is the probability to find the bare one-photon state in the physical one-photon state of vanishing momentum, divided by the probability to find the bare vacuum in the physical vacuum.

1. Introduction. The aim of this paper is to present an answer to a simple question in the quantum theory of interacting fields: what does one find when one tries to express by perturbation methods the exact stationary states of the interacting fields in terms of the free-field eigenstates? That comparatively little attention has been paid to this question is completely in line with the philosophy of present-day field theory, which avoids as much as possible the use of the unobservable free-field variables and states. Either by the artifice of switching on and off the interaction, or by exclusive consideration of the physical (i.e. dressed) vacuum and particle states, one avoids the necessity of knowing the exact expression of these physical, observable states in terms of the unphysical free-field (bare) states. Although we do not mean to doubt the soundness of this standpoint, we nevertheless believe that the structure of the physical states in terms of the bare states is worth investigating. After all, the occurrence in the hamiltonian of an interaction term of specific algebraic form, although not a directly observable feature, is in an indirect way, at least in electrodynamics, imposed upon us by the empirical facts. In addition, all present day formalisms implicitly or explicitly imply the simultaneous presence of the physical particles and

*) Predoctoral Fellow of the U.S. National Science Foundation. Present address: Department of Physics, University of California, Berkeley, California. 
the unobservable bare particles, the latter being unavoidably brought in by the field operators, even when taken in the renormalized form.

Our problem will be solved by perturbation expansion, under the assumption that ultra-violet divergences are avoided by introduction of a cut-off momentum $K$ and infra-red divergences by non-vanishing mass values for all particles. A solution is directly obtained by application of an improved version of time-independent perturbation theory, developped in earlier papers ${ }^{1}$ ) (quoted hereafter as I, II and III) by Hugenholt $z$ and one of the present authors. This perturbation method, meant to apply in general to systems with many degrees of freedom; was developped mainly for the study of many particle systems like solids and gases, i.e. non-relativistic systems. Its application to interacting fields is immediate, but it is formally non-covariant. This circumstance is no difficulty for our aim, which is of general nature. It is also convenient when discussing the non-relativistic simplified models of field theory to which so much attention has been devoted lately. For actual computations on a fully relativistic theory, however, it is clear that a covariant reformulation of our method would be desirable.

The general features of the method and the corresponding diagram technique are outlined in the two next sections. We then give in section 4 the explicit expression for the stationary states of a system of.interacting fields, treating separately the vacuum, the one-particle states and the states with more than one particle, which manifest collision processes (scattering, creation and annihilation). Section 4 also studies the relation between the states with two or more particles and the one-particle states. This relation, which is the most important observable element of the theory, is expressed by the $S$-matrix. A few points of comparison between our method and the conventional covariant formalism are discussed in Section 5 . They concern mainly the renormalization constants $Z_{2}$ and $Z_{3}$ of electron and photon (we use Dyson's notation ${ }^{2}$ ) for these constants). Whereas the former is replaced in our formalism by another quantity $N_{k}$, the constant $Z_{3}$ (the square root of which is the ratio of renormalized to unrenormalized charge) is retained but receives a new physical interpretation: it is found to be the probability of finding the bare photon in the physical photon state of momentum zero, divided by the probability of finding the bare vacuum in the physical vacuum state.

2. The resolvent operator and its relation to the eigenstates. To simplify the notation we consider the case of a scaler neutral Klein-Gordon field $A$ with an interaction density $g A^{3}$. We work in a given Lorentz frame and write the total hamiltonian $H=H_{0}+V$ in the Schrödinger representation, in terms of creation and annihiliation operators for free particles, as the sum of the free energy term

$$
H_{0}=\sum_{k} \varepsilon_{k} a_{k}^{*} a_{k}
$$


and the interaction term

$$
V=g(8 \Omega)^{-t} \sum_{k k^{\prime}}\left(\varepsilon_{k^{\prime}} \varepsilon_{k^{\prime}} \varepsilon_{k+k^{\prime}}\right)^{-\xi}\left(a_{k}+a_{-k}^{*}\right)\left(a_{k^{\prime}}+a_{-k^{\prime}}^{*}\right)\left(a_{-k-k^{\prime}}+a_{k+k^{\prime}}^{*}\right) \text {. }
$$

$k, k^{\prime}$ denote the momentum vectors of the free particles. The system is enclosed in a cubic box of volume $\Omega$ and periodic boundary conditions are used. The vectors $k, k^{\prime}$ are quantized accordingly. $\varepsilon_{k}$ is the energy $\left(|k|^{2}+\mu^{2}\right)^{1}$ where $\mu$ is the non-renormalized mass and the units have been chosen so that $\hbar=c=1$. The creation and annihilation operators satisfy the usual commutation rules

$$
\left[a_{k}, a_{k^{\prime}}\right]=0,\left[a_{k}, a_{k^{\prime}}^{*}\right]=\delta_{k k^{\prime}} .
$$

In the interaction (2.2) the summation is restricted to $|k|<K,\left|k^{\prime}\right|<K$, $K$ being the cut- off.

As a complete set of eigenstates of $H_{0}$ we choose the vectors

$$
|\alpha\rangle=\left|k_{1} \ldots k_{n}\right\rangle=\left(\Omega / 8 \pi^{3}\right)^{n / 2} a_{k_{1}}^{*} \ldots a_{k_{n}}^{*}|0\rangle,
$$

$|0\rangle$ being the normalized unperturbed vacuum!state. Adopting the convention of writing the $n$ vectors $k_{1} \ldots k_{n}$ in a prescribed order, we find when all $k_{i}$ are different

$$
\left\langle k_{1} \ldots k_{n} \mid k_{1}^{\prime} \ldots k_{n^{\prime}}^{\prime}\right\rangle=\delta_{n n^{\prime}} \delta\left(k_{1}-k_{1}^{\prime}\right) \ldots \delta\left(k_{n}-k_{n}^{\prime}\right) .
$$

Here the symbol $\delta(k)$ stands for

$$
\delta(k)=0 \text { for } k \neq 0, \delta(k)=\Omega / 8 \pi^{3} \text { for } k=0 .
$$

It reduces to a three-dimensional $\delta$-function in the limiting case of large $\Omega$, the only case of interest. The normalization thus adopted for the states $|\alpha\rangle$ is such that the matrix elements of $V$ become independent of $\Omega$ if momentum conservation is expressed by means of a $\delta$-function (2.6). For instance

$$
\left\langle k_{1} k_{2}|V| k\right\rangle=\left(3 g / 4 \pi^{3 / 2}\right) \delta\left(k_{1}+k_{2}-k\right) .
$$

Also summations over intermediate states take a simple form when use is made of the relation

$$
\left(8 \pi^{3} / \Omega\right)^{n} \sum_{k \ldots k_{n}}=\int \mathrm{d} k_{1} \ldots \mathrm{d} k_{n} \quad \text { for large } \Omega .
$$

For instance, $\mathrm{O}_{1}$ and $\mathrm{O}_{2}$ being. two operators,

$$
\begin{aligned}
& \left\langle\alpha\left|\mathrm{O}_{1} \mathrm{O}_{2}\right| \alpha^{\prime}\right\rangle=\sum_{n} \int \mathrm{d} k_{1} \ldots \mathrm{d} k_{n}\left\langle\alpha\left|\mathrm{O}_{1}\right| k_{1} \ldots k_{n}\right\rangle . \\
& \left.\cdot-k_{1} \ldots k_{n}\left|\mathrm{O}_{2}\right| \alpha^{\prime}\right\rangle=\int \mathrm{d} \alpha^{\prime \prime}\left\langle\alpha\left|\mathrm{O}_{1}\right| \alpha^{\prime \prime}\right\rangle\left\langle\alpha^{\prime \prime}\left|\mathrm{O}_{2}\right| \alpha^{\prime}\right\rangle .
\end{aligned}
$$

One should keep in mind that all $k$ 's denote three-dimensional vectors. Each integration $\int \mathrm{d} k$ is also three-dimensional.

The perturbation method used in I, II, III and in the present paper is based on the resolvent operator defined by

$$
R_{l}=(H-l)^{-1}
$$


for complex $l$. This operator is the complex Fourier transform of the unitary operator of motion

$$
U_{t}=\exp (-i H t)
$$

Our method is in essence the complex Fourier transform of the usual timedependent perturbation theory. For fields the latter formalism starts from the straight perturbation expansion of (2.11) and carries out a number of partial summations exactly. Our method does the same for the resolvent (2.10). Its advantage for our present purpose lies in the fact that the eigenstates of $H$ are more simply related to the resolvent than to the operator of motion.

When one calculates a matrix element

$$
\left\langle\alpha\left|R_{l}\right| \alpha^{\prime}\right\rangle=\left\langle k_{1} \ldots k_{n}\left|R_{l}\right| k_{1}^{\prime} \ldots k_{n^{\prime}}^{\prime}\right\rangle
$$

to any order in the perturbation and studies its dependence on the variables $k_{1}, \ldots k_{n^{\prime}}^{\prime}$, it is found to contain terms with a maximal $\delta$-singularity

$$
\delta_{n n^{\prime}} \delta\left(k_{1}-k_{1}^{\prime}\right) \ldots \delta\left(k_{n}-k_{n}^{\prime}\right)
$$

as well as terms with weaker $\delta$-singularities, i.e. terms where at least one of the states $\alpha, \alpha^{\prime}$ is not entirely determined by the other $\left.{ }^{*}\right)$. The sum of all terms with the maximal singularity (2.13) we denote by

$$
D_{l}\left(k_{1} \ldots k_{n}\right) \delta_{n n^{\prime}} \delta\left(k_{1}-k_{1}^{\prime}\right) \ldots \delta\left(k_{n}-k_{n}^{\prime}\right)
$$

or more briefly by

$$
D_{l}(\alpha) \delta\left(\alpha-\alpha^{\prime}\right) .
$$

It is called the diagonal part ${ }^{* *}$ ) of the matrix element (2.12). We extend this definition to the unperturbed vacuum state $|0\rangle$ by putting

$$
D_{l}(0)=\left\langle 0\left|R_{l}\right| 0\right\rangle \text {. }
$$

The diagonal operator $D_{l}$ defined by

$$
D_{l}|\alpha\rangle=|\alpha\rangle D_{l}(\alpha)
$$

is called the diagonal part of $R_{l}$. The analytical behaviour of $D_{l}(\alpha)$ as a function of the complex number $l$ was studied in I for the limiting case of large $\Omega$. For interacting fields $D_{l}(\alpha)$ has for each state $\alpha$ a simple pole in a point $l=E(\alpha)$ of the real axis, with a residue $N(\alpha)$ verifying the inequalities

$$
0<N(\alpha)<1 \text {. }
$$

Its further singularities cover the portion of the real axis from a point $E^{\prime}(\alpha)>E(\alpha)$ to $+\infty$. In each point of this interval $D_{l}(\alpha)$ has a finite

*) By $\delta$-singularities we understand quantities of type (2.6) which are $\delta$-functions in the limit of large $\Omega$.

**) By diagonal we shall always mean diagonal in the representation (2.4). 
discontinuity for $l$ crossing the real axis; the discontinuity of the function amounts to a change of sign of its imaginary part *).

We quote now the main result of II (section 2). It holds under the assumptions of large $\Omega$ and of convergence of all series involved; these assumptions are necessary throughout the present paper and will not be repeated. To each eigenstate $|\alpha\rangle$ of $H_{0}$ there correspond two normalized eigenstates of the total hamiltonian $H$, to be denoted by $|\alpha\rangle_{ \pm}$. Both have $E(\alpha)$ as corresponding eigenvalue of $H$. Their expression is

$$
\mid \alpha)_{ \pm}=[N(\alpha)]^{k} \cdot\left[1+\sum_{s=1}^{\infty}\left\{\left(-D_{E(\alpha) \pm i 0}^{0} V\right)^{s}\right\}_{n i}\right]|\alpha\rangle .
$$

This equation is identical with eq. (2.7) of II, but is expressed with the unperturbed resolvent

$$
D_{l}^{0}=\left(H_{0}-l\right)^{-1} .
$$

Upper (lower) signs must be taken together in (2.18) and in later formulae. The symbol $\left\{\left(D_{l}^{0} V\right)^{s}\right\}_{n i}$, where the subscript is an abbreviation for noninitial state, means that the matrix element

$$
\begin{array}{r}
\left\langle\alpha^{\prime}\left|\left(D_{l}^{0} V\right)^{s}\right| \alpha\right\rangle=D_{l}^{0}\left(\alpha^{\prime}\right) \int\left\langle\alpha^{\prime}|V| \alpha_{s-1}\right\rangle D_{l}^{0}\left(\alpha_{s-1}\right) \mathrm{d} \alpha_{s-1} \ldots \\
\ldots\left\langle\alpha_{2}|V| \alpha_{1}\right\rangle D_{l}^{0}\left(\alpha_{1}\right) \mathrm{d} \alpha_{1}\left\langle\alpha_{1}|\mathrm{~V}| \alpha\right\rangle
\end{array}
$$

is replaced by zero for $\alpha^{\prime}=\alpha$ and is evaluated for $\alpha^{\prime} \neq \alpha$ by restricting the integration to intermediate states different from $\alpha$. This restriction insures that (2.20) remains finite for $l=E(\alpha) \pm i 0$. The states $|\alpha\rangle_{ \pm}$are normalized in the same way as the unperturbed states $|\alpha\rangle$ :

or more explicitly

$$
{ }_{+}\left(\alpha \mid \alpha^{\prime}\right)_{+}={ }_{-}\left(\alpha \mid \alpha^{\prime}\right)_{-}=\delta\left(\alpha-\alpha^{\prime}\right)
$$

$$
{ }_{ \pm}\left(k_{1} \ldots k_{n} \mid k_{1}^{\prime} \ldots k_{n^{\prime}}^{\prime}\right)_{ \pm}=\delta_{n n^{\prime}} \delta\left(k_{1}-k_{1}^{\prime}\right) \ldots \delta\left(k_{n}-k_{n}^{\prime}\right) .
$$

As will be seen later (section 4$), \mid \alpha)_{+}$corresponds to a state where all scattered waves are outgoing, $\mid \alpha)_{-}$to a state where they are incoming waves. An alternative expression of (2.18) is

$$
|\alpha\rangle_{ \pm}=[N(\alpha)]^{-\frac{t}{t}} \lim _{l \rightarrow E(\alpha)}[E(\alpha)-l] R_{l}|\alpha\rangle \text { for } \pm \operatorname{Im}(l)>0 .
$$

3. The diagrams. The matrix elements of the resolvent have been analyzed in terms of diagrams in III (the complex variable $l$ is there denoted bij $z$ ). Consider the perturbation expansion of $R_{l}$ :

$$
R_{l}=D_{l}^{0}-D_{l}^{0} V D_{l}^{0}+D_{l}^{0} V_{l}^{0} D_{l}^{0} V D_{l}^{0}-\ldots
$$

*) It is through the analytical singularities of $D_{l}(\alpha)$ that the physical difference between fields and many-particle systems manifests itself in the formalism. Except in their ground state manyparticle systems have an essentially dissipative behaviour. This situation reflects itself mathematically by the absence of poles in $D_{l}(\alpha)$. A detailed consideration of dissipative systems has been Idesented elsewhere ${ }^{3}$ ). 
and take for a matrix element of an arbitrary term

$$
\left\langle\alpha^{\prime}\left|D_{l}^{0} V D_{l}^{0} V \ldots D_{l}^{0} V D_{l}^{0}\right| \alpha\right\rangle
$$

a possible succession of intermediate states $\left|\alpha_{1}\right\rangle,\left|\alpha_{2}\right\rangle, \ldots$ leading from $|\alpha\rangle$ to $\left|\alpha^{\prime}\right\rangle$ by transitions caused by the various operators $V$. Such a transition scheme is represented by a diagram: to each operator $V$ corresponds a point (vertex), to each of the states $\alpha, \alpha_{1}, \alpha_{2} \ldots \alpha^{\prime}$ a set of lines directed more or less horizontally, one line for each particle present in the state. There is no line in the case of the vacuum state $|0\rangle$. If a particle is involved in the transition caused by an operator $V$, its representing line ends in the vertex corresponding to this $V$. Otherwise its line is continued without interruption from state to state. The succession of sets of lines and vertices in the diagram, taken from right to left, corresponds to the right to left succession of states and operators $V$ in the transition scheme of the matrix element. For example, the diagram of fig. 1 corresponds to the transition scheme

$$
\left|\alpha^{\prime}\right\rangle=\left|k_{1}^{\prime} k_{2}^{\prime}\right\rangle,\left|\alpha_{1}\right\rangle=|k\rangle,|\alpha\rangle=\left|k_{1} k_{2}\right\rangle
$$

belonging to the second order term $\left\langle\alpha^{\prime}\left|D_{l}^{0} V D_{l}^{0} V D_{l}^{0}\right| \alpha\right\rangle$ of $\left\langle\alpha^{\prime}\left|R_{l}\right| \alpha\right\rangle$, whereas the following transition scheme, belonging to (3.2) with four $V$ operators,

$$
\begin{aligned}
& \left|\alpha^{\prime}\right\rangle=\left|k_{1} k_{2}^{\prime}\right\rangle,\left|\alpha_{3}\right\rangle=\left|k_{1} k_{2}^{\prime} k k^{\prime} k^{\prime \prime}\right\rangle,\left|\alpha_{2}\right\rangle=\left|k_{1} k_{2}^{\prime \prime} k_{2}^{\prime \prime \prime} k k^{\prime} k^{\prime \prime}\right\rangle, \\
& \left|\alpha_{1}\right\rangle=\left|k_{1} k_{2}^{\prime \prime} k_{2}^{\prime \prime \prime}\right\rangle,|\alpha\rangle=\left|k_{1} k_{2}\right\rangle,
\end{aligned}
$$

is represented by the diagram of fig. 2. The order of a diagram is defined as its number of vertices; it is equal to the order in $V$ of the corresponding contribution to $\left\langle\alpha^{\prime}\left|R_{l}\right| \alpha\right\rangle$.
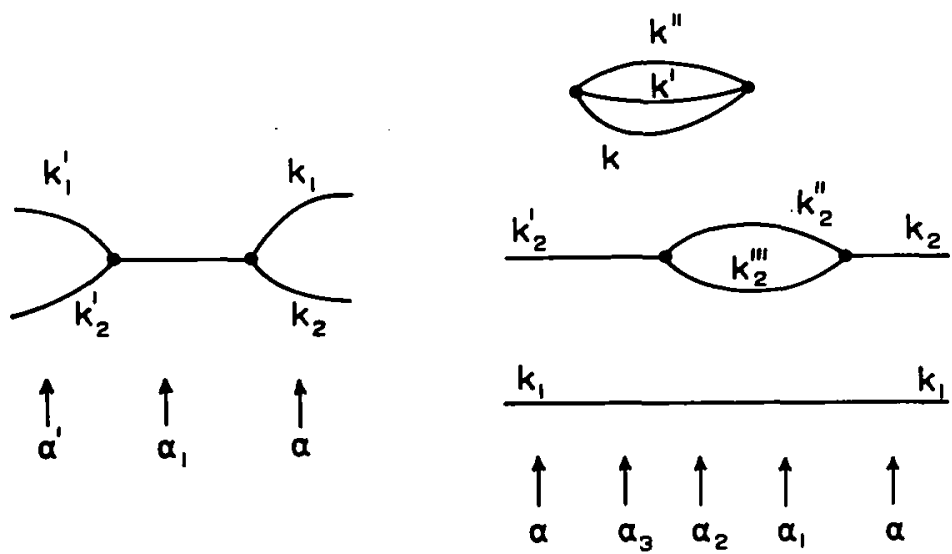

Fig. 1 An example of diagram of second order.

Fig. 2. A diagram of order four.

The diagrams here defined differ in one respect only from the Feynman diagrams usually considered in covariant field theory. For our diagrams 
the relative positions of the vertices from right to left are significant; two diagrams differing by the ordering of vertices describe different transition schemes and consequently give rise to different contributions to the resolvent. This distinction can, however, be lifted to a certain extent for practical calculations. The definition of our diagrams for other types of fields and interactions is obvious. We mention only that for a Fermi-Dirac field each particle line gets an orientation indicated by an arrow: lines pointing to the left represent particles and lines pointing to the right antiparticles. The number of fermion lines ending in a vertex is always even, half of them being oriented toward the vertex and the other half away from it (see III).

A diagram is connected when one can go from any of its lines to any other by continuous displacement on the diagram, along its lines and through its vertices. A non-connected diagram is always formed by juxtaposition of a number of connected diagrams, which we shall call its components. More generally we call any connected diagram a component. The diagram of fig. 1 is connected, the diagram of fig. 2 is non-connected and has three components. One should note that the various components of a non-connected diagram are not located arbitrarily with respect to each other; there is a prescribed

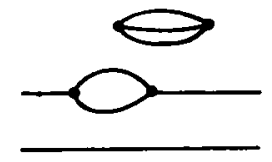

$\left(\delta^{\prime}\right)$

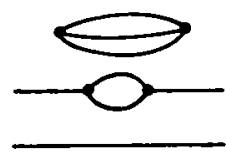

(6")
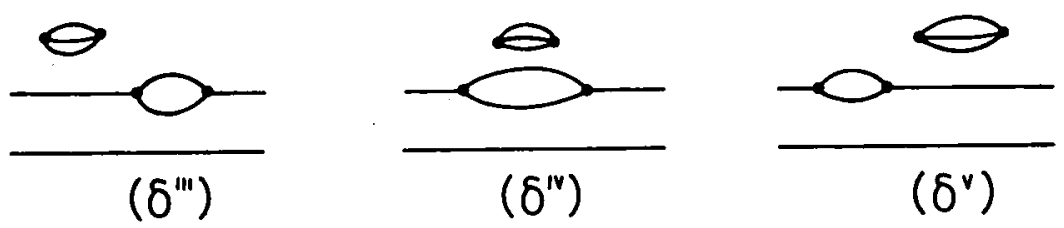

Fig. 3. The diagrams obtained from the diagram of fig. 2 by displacement of the various components.

right to left ordering of all vertices, even those belonging to different components. Consider, however, for a given non-connected diagram $\delta$, all nonconnected diagrams $\delta^{\prime}, \delta^{\prime \prime}, \ldots$ composed of the same components $\delta_{1}, \delta_{2}, \ldots$ as $\delta$, but with other orderings of the vertices of different components (the mutual ordering of vertices inside each component individually is of course kept unchanged. For example, if $\delta$ is the diagram of fig. 2, the diagrams $\delta^{\prime}, \delta^{\prime \prime}, \ldots$ are those of fig. 3). As shown in III (section 7) there is a simple formula expressing the total contribution of the diagrams $\delta, \delta^{\prime}, \delta^{\prime \prime} \ldots$ to the 
resolvent in terms of the contributions of $\delta_{1}, \delta_{2}, \ldots$ :

$$
\begin{aligned}
\left\langle\alpha^{\prime}\left|\left\{R_{l}\right\}_{\delta}\right| \alpha\right\rangle+\left\langle\alpha^{\prime}\left|\left\{R_{l}\right\}_{\delta^{\prime}}\right| \alpha\right\rangle & +\left\langle\alpha^{\prime}\left|\left\{R_{l}\right\}_{\delta^{\prime \prime}}\right| \alpha\right\rangle+\ldots \\
& =\left\langle\alpha_{1}^{\prime}\left|\left\{R_{l}\right\}_{\delta_{1}}\right| \alpha_{1}\right\rangle *\left\langle\alpha_{2}^{\prime}\left|\left\{R_{l}\right\}_{\delta_{2}}\right| \alpha_{2}\right\rangle * \ldots
\end{aligned}
$$

The notation $\left\langle\beta^{\prime}\left|\left\{R_{l}\right\}_{\gamma}\right| \beta\right\rangle$ for a diagram $\gamma$ denotes the contribution of the diagram to the matrix element $\left\langle\beta^{\prime}\left|R_{l}\right| \beta\right\rangle, \beta$ and $\beta^{\prime}$ being respectively the initial and final states represented at the extreme right and extreme left of the diagram. The operation $*$ is a convolution in the complex plane. For any two functions $f_{l}, g_{l}$ which are holomorphic for $l$ non-real and decrease at least as $|l|^{-1}$ for $l \rightarrow \infty$, the convolution is defined as

$$
f_{l} * g_{l}=(i / 2 \pi) \int \mathrm{d} l^{\prime} f_{l-l^{\prime}} g_{l^{\prime}} \text { for } \operatorname{Im}(l) \neq 0 .
$$

The path of integration must encircle once counterclockwise all points where $g_{l^{\prime}}$ is singular but may not cross the line $\operatorname{Im}(l)=\operatorname{Im}\left(l^{\prime}\right)$. The product $f_{l} * g_{l}$ is an analytic function with all its singularities on the real axis. It is symmetric in the factors $f_{l}, g_{l}$. For three or more factors the convolution product is associative.

The validity of the relation (3.3) requires that an important rule be followed in calculating the contributions of diagrams to the resolvent. The calculation should always be carried out as if the particles represented by the various lines of the diagram were in different one-particle states. Corrections must never be introduced for the exceptional cases where two or more identical particles would be in the same state. It is well known ${ }^{4}$ ) that such corrections always exactly cancel out against each other. If introduced, however, they would destroy the validity of (3.3) and render impossible the all-important reduction to connected diagrams. The same remark is known to apply to the conventional covariant formalism of field theory.

An external line of a diagram is a line which does not join two vertices; such a line necessarily extends either to the extreme right of the diagram, or to the extreme left, or to both. A diagram, all the components of which have external lines, gives to $R_{l}$ a contribution not explicitly dependent on $\Omega$ for large $\Omega$ (implicitly there is an $\Omega$-dependence through $\delta$-functions). Any other diagram gives a contribution proportional to $\Omega^{\nu}$ if $v$ is the number of its components without external lines (III, section 6). A diagram (or component) without external lines will be called vacuum diagram (or vacuum component), because it contributes to the vacuum matrix element $\left\langle 0\left|R_{l}\right| 0\right\rangle$. A one-particle diagram is a diagram with exactly two external lines, one ending at the extreme right and one at the extreme left. If such a diagram is connected we call it a one-particle component. By momentum of a one-particle diagram or component we mean the momentum of the particle described by the external lines. Examples of vacuum and one- 
particle components are given in fig. 4 and 5 respectively. As will now be seen, the diagonal part $D_{l}$ of the resolvent is entirely expressible in terms of vacuum and one-particle components.

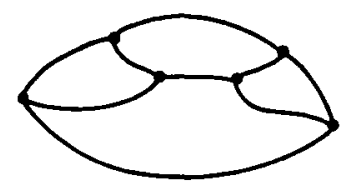

Fig. 4. An example of vacuum component.

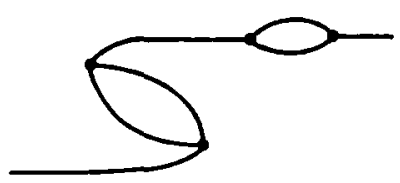

Fig. 5. An example of one-particle component.

Consider first the function

$$
\Sigma_{\delta}\left\langle 0\left|\left\{R_{l}\right\}_{\delta}\right| 0\right\rangle=B_{l}=l^{-2} \Omega F_{l},
$$

where the summation extends over all vacuum components $\delta$. As shown in III (sections 6, 8 and 9; the function $\Omega F_{l}$ is there written $\bar{G}_{0}(z)$; the quantity $\varepsilon_{0}$ of III is here zero), the function $F_{l}$ has a finite limit for $\Omega \rightarrow \infty$ and its only singularities are finite discontinuities accross the real axis for all real values of $l$ larger than a positive number $E_{0}^{\prime}$. Since the most general vacuum diagram is a juxtaposition of vacuum components, application of (3.3) gives for the value $D_{l}(0)$ of $D_{l}$ for the vacuum state

$$
D_{l}(0)=-l^{-1}+B_{l}+\frac{1}{2}\left(B_{l} * B_{l}\right)+(3 !)^{-1}\left(B_{l} * B_{l} * B_{l}\right)+\ldots
$$

One of the most important results of III was to show that the pole $E(0)$ and the residue $N(0)$ of $D_{l}(0)$ are given by

$$
E(0)=-\Omega F_{0}, N(0)=\exp \left(-\Omega F_{0}^{\prime}\right),
$$

where $F_{0}$ and $F_{0}^{\prime}$ are the values of $F_{l}$ and $\mathrm{d} F_{l} / \mathrm{d} l$ at $l=0 . F_{0}^{\prime}$ is non-negative (see III, section 9).

Consider next the function

$$
\Sigma_{\delta}\left\langle k^{\prime}\left|\left\{R_{l}\right\}_{\delta}\right| k\right\rangle=C_{l}(k) \delta\left(k^{\prime}-k\right)
$$

with the summation extending over all one-particle components of momentum $k . C_{l}(k)$ taken for large $\Omega$ is independent of $\Omega$, has a simple pole in a point $l=E_{k}$ of the real axis, with a real positive residue $N_{k}$, and has finite discontinuities across the real axis for $l$ real and larger than a number $E_{k}^{\prime}>E_{k}$ (see III, section 10 , where $C_{l}(k), E_{k}$ and $N_{k}$ are denoted by $\bar{D}_{\alpha}(z)$, $\bar{E}_{\alpha}$ and $\bar{N}_{\alpha}$ for $\alpha=k$ ). Each one-particle diagram is composed of a oneparticle component and possibly one or more vacuum components. (3.3) gives accordingly

$$
D_{l}(k)=D_{l}(0) * C_{l}(k) .
$$


This relation directly determines the pole $E(k)$ and residue $N(k)$ of $D_{l}(k)$ :

$$
\begin{aligned}
& E(k)=E(0)+E_{k}=-\Omega F_{0}+E_{k}, \\
& N(k)=N(0) N_{k}=\exp \left(-\Omega F_{0}^{\prime}\right) N_{k} .
\end{aligned}
$$

If one considers finally states $|\alpha\rangle=\left|k_{1} \ldots k_{n}\right\rangle$ with more than one particle, one easily verifies that the only diagrams contributing to the diagonal part (2.14) of the matrix element (2.12) are formed by juxtaposition of $n$ oneparticle components, of momenta $k_{1}, \ldots k_{n}$, with possibly in addition some vacuum components. This implies immediately

$$
\begin{aligned}
& D_{l}\left(k_{1} \ldots k_{n}\right)=D_{l}(0) * C_{l}\left(k_{1}\right) * \ldots * C_{l}\left(k_{n}\right), \\
& E\left(k_{1} \ldots k_{n}\right)=E(0)+E_{k_{1}}+\ldots+E_{k_{n}}, \\
& N\left(k_{1} \ldots k_{n}\right)=N(0) N_{k_{1}} \ldots N_{k_{n^{*}}}
\end{aligned}
$$

4. The stationary states and the S-matrix. As shown in III, a diagram analysis of the stationary states can be carried out on the basis of equations (2.18) or (2.23). We mention first the result for the vacuum state. We must for convenience redefine slightly the creation and annihiliation operators for free particles:

$$
A_{k}=\left(\Omega / 8 \pi^{3}\right)^{\frac{1}{2}} a_{k}, A_{k}^{*}=\left(\Omega / 8 \pi^{3}\right)^{\frac{1}{2}} a_{k}^{*} .
$$

The commutation rule is now, from (2.3) and (2.6),

and one has

$$
\left[A_{k}, A_{k^{\prime}}^{*}\right]=\delta\left(k-k^{\prime}\right),
$$

$$
\left.\left|k_{1} \ldots k_{n}>=A_{k_{1}}^{*} \ldots A_{k_{n}}^{*}\right| 0\right\rangle \text {. }
$$

Consider the operator

$$
0_{0}=-\lim _{l \rightarrow 0} l \Sigma_{n>0} \int \mathrm{d} k_{1} \ldots \mathrm{d} k_{n} \Sigma_{\gamma}\left\langle k_{1} \ldots k_{n}\left|\left\{R_{l}\right\}_{\gamma}\right| 0\right\rangle A_{k_{1}}^{*} \ldots A_{k_{n}}^{*}
$$

where the second sum extends over connected diagrams $\gamma$. The value of $O_{0}$ is independent of the way $l$ tends to zero and it can be written alternatively

$$
0_{0}=-\Sigma_{n>0} \int \mathrm{d} k_{1} \ldots \mathrm{d} k_{n} \Sigma_{\gamma}\left\langle k_{1} \ldots k_{n}\left|\left\{R_{0} V\right\}_{\gamma}\right| 0\right\rangle A_{k_{1}}^{*} \ldots A_{k_{n}}^{*} .
$$

Application of (2.18) gives a common value to $(0)_{+}$and $(0)_{-}$. Denoting this state, the vacuum state of the total hamiltonian, by $(0)$, one finds

$$
\begin{aligned}
\mid 0) & =[N(0)]^{\ddagger}\left(1+0_{0}+0_{0}^{2} / 2 !+\ldots\right)|0\rangle \\
& =\exp \left[-\frac{1}{2} \Omega F_{0}^{\prime}+0_{0}\right]|0\rangle .
\end{aligned}
$$

This simple result has been established in III, section 11 . The coefficient $N(0)=\exp \left[-\Omega F_{0}^{\prime}\right]$ has an obvious physical meaning: it is the probability of finding the unperturbed vacuum $|0\rangle$ in the physical vacuum $\mid 0)$. The exponential dependence of this quantity on $\Omega$ is a manifestation of the fact that the interaction $V$ modifies the vacuum state homogeneously over the whole extension of the volume $\Omega$. The same fact exhibits itself through the 
proportionality to $\Omega$ of the vacuum energy $E(0)=-\Omega F_{0}$, as well as thro the exponential occurrence in (4.6) of the operator $0_{0}$ which involves $c$ nected diagrams only.

Also the physical one-particle states $|k\rangle_{ \pm}$are found to be independen the double sign, as is expected physically. Putting $|k|_{ \pm}=|k|$ one obte from (2.23) (see section 12 of III)

with

$$
\left.\mid k)=0_{k} \mid 0\right)
$$

$$
\begin{aligned}
\mathrm{O}_{k}=N_{k}^{\frac{1}{k}}\left[A_{k}^{*}-\right. & N_{k}^{-1} \lim _{l \rightarrow E_{k}}\left(l-E_{k}\right) . \\
& \cdot \Sigma_{n>1} \int \mathrm{d} k_{1} \ldots \mathrm{d} k_{n} \Sigma_{\gamma}\left\langle k_{1} \ldots k_{n}\right|\left\{R_{l\}_{\gamma}}|k\rangle A_{k_{1}}^{*} \ldots A_{k_{n}}^{*}\right] .
\end{aligned}
$$

The second summation extends over connected diagrams. The limit is dependent of the way $l$ approaches $E_{k}$. One can write alternatively.

$0_{k}=N_{k}^{t}\left[A_{k}^{*}-\Sigma_{n>1} \int \mathrm{d} k_{1} \ldots \mathrm{d} k_{n} \Sigma_{\delta}\left\langle k_{1} \ldots k_{n}\left|\left\{R_{E_{k}} V\right\}_{\delta}\right| k\right\rangle A_{k_{1}}^{*} \ldots A_{k_{n}}^{*}\right],(4$

the $\delta$ 's being now connected diagrams with more than one particle in e: intermediate state. The interest of equation (4.7) does of course not lie the mere existence of an operator $O_{k}$ verifying it: many such operators c be found. It is rather that one such operator has a very simple express: in terms of connected diagrams. The energy of the state $\mid k$ ) is given by (3.1 The observable part of this energy is $E_{k}=E(k)-E(0)$, the excitat energy of the physical particle state above the physical vacuum state. ] significance of $N_{k}$ is also clear. From (3.11) it is the probability $N(k)$ finding the bare particle state $|k\rangle$ in the physical particle state $|k\rangle$, divic by the probability $N(0)$ to find the bare vacuum in the physical vacuu $N_{k}$ is positive. It is smaller than one when $N(0)=1$, i.e. for such int actions which do not modify the vacuum state: $|0|=|0\rangle$.

We now give the expression of the $n$-particle stationary states $\mid k_{1} \ldots k$ for $n \geq 2$. They describe the scattering, annihilation and creation proces produced by the interaction, and a distinction must be made between 1 state with outgoing scattered waves (upper sign) and the state with incomi scattered waves (lower sign).

One finds

$$
\left.\left.\mid k_{1} \ldots k_{n}\right)_{ \pm}=0_{ \pm}\left(k_{1} \ldots k_{n}\right) \mid 0\right)
$$

with

$0_{ \pm}\left(k_{1} \ldots k_{n}\right)=\left(N_{k_{1}} \ldots N_{k_{n}}\right)^{\frac{1}{2}}\left[A_{k_{1}}^{*} \ldots A_{k_{n}}^{*}-\left(N_{k_{1}} \ldots N_{k_{n}}\right)^{-1}\right.$.

. $\left.\lim \left(l-E_{k_{1}}-\ldots E_{k_{n}}\right) \sum_{n^{\prime}} \int \mathrm{d} k_{1}^{\prime} \ldots \mathrm{d} k_{n^{\prime}}^{\prime}\left\langle k_{1}^{\prime} \ldots k_{n^{\prime}}^{\prime}\left|\left\{R_{l}\right\}_{\gamma}\right| k_{1} \ldots k_{n}\right\rangle A_{k_{1}}^{*} \ldots A_{k^{\prime} n^{\prime}}^{*}\right]$ (4.

where the limit is taken for $l \rightarrow E_{k_{1}}+\ldots E_{k_{n}}$ with $\pm \operatorname{Im}(l)>0$. The are diagrams without vacuum components, not entirely composed 
one-particle components. Again we can write equivalently, putting $E=E_{k_{1}}+\ldots E_{k_{\mathrm{n}}}$,

$$
\begin{aligned}
& O_{ \pm}\left(k_{1} \ldots k_{n}\right)=\left(N_{k_{1}} \ldots N_{k_{n}}\right)^{ \pm}\left[A_{k_{1}}^{*} \ldots A_{k_{n}}^{*}-\right. \\
& \left.\quad \sum n^{\prime} \int \mathrm{d} k_{1}^{\prime} \ldots \mathrm{d} k_{n^{\prime}}^{\prime} \Sigma_{\delta}\left\langle k_{1}^{\prime} \ldots k_{n^{\prime}}^{\prime}\left|\left\{R_{E \pm i 0} V\right\}_{\delta}\right| k_{1} \ldots k_{n}\right\rangle A_{k_{1}^{\prime}}^{*} \ldots A_{k^{\prime} n^{\prime}}^{*}\right] .
\end{aligned}
$$

Here the $\delta$ 's are diagrams without vacuum components satisfying the further condition that neither the diagram itself, nor the part of it on the right of any intermediate state is composed of $n$ one-particle components. The energy of the states $\left|k_{1} \ldots k_{n}\right\rangle_{ \pm}$is given by (3.13); its observable part, i.e. the excitation energy above the physical vacuum, is the sum $E_{k_{1}}+\ldots E_{k_{n}}$ of the excitation energies of the $n$ particles characterizing the state.

By classifying in (4.11) the contributing diagrams according to connectedness, one can further decompose the operator $0_{ \pm}\left(k_{1} \ldots k_{n}\right)$ in parts which correspond to binary, ternary, ... n-particle interactions, according to which groups of the incoming (for the upper sign) or outgoing (for the lower sign) particles actually react with each other. Let us define the operators $0_{ \pm}^{c}\left(k_{1} \ldots k_{n}\right)$ inductively by the equations

$$
\begin{gathered}
O_{ \pm}^{c}(k)=0_{k} . \\
O_{ \pm}\left(k_{1} \ldots k_{n}\right)=0_{ \pm}^{c}\left(k_{1} \ldots k_{n}\right)+\Sigma 0_{ \pm}^{c}\left(k_{f} k_{j^{\prime}} \ldots\right) O_{ \pm}^{c}\left(k_{n} k_{h^{\prime}} \ldots\right) \ldots,
\end{gathered}
$$

where the sum extends over all distinct ways of grouping the momenta $k_{1}, \ldots k_{n}$ in two or more subgroups of one or more elements. The expression of the operator $O_{ \pm}^{c}\left(k_{1} \ldots k_{n}\right)$ in terms of diagrams is easily obtained: it differs from (4.11) and (4.12) by omission of the first term in the square brackets and by the additional restriction that the diagrams summed over in the second term be connected (this explains the index $c$ in $0_{ \pm}^{c}$ ). As easily seen by considering the motion of wave packets, the operator $0_{+}^{c}\left(k_{1}, \ldots, k_{n}\right)$ corresponds to the portion of the stationary state $\left|k_{1} \ldots k_{n}\right\rangle_{+}$where all incoming particles interact with each other, whereas the other terms in the righthand side of (4.14) describe processes where groups of incoming particles remain separated from each other. A similar interpretation holds for $0_{-}^{c}$.

To relate the formula (4.10) to the main observable features of a collision experiment, let us consider a general time dependent wave function

$$
\left.\varphi_{ \pm}(t)=\int \mid k_{1} \ldots k_{n}\right)_{ \pm} \mathrm{d} k_{1} \ldots \mathrm{d} k_{n} \exp \left[-i\left(E_{k_{1}}+\ldots E_{k_{n}}\right) t\right] c\left(k_{1} \ldots k_{n}\right),
$$

solution of the Schrödinger equation

$$
i \partial \varphi_{ \pm}(t) / \partial t=[H-E(0)] \varphi_{ \pm}(t)
$$

where the unobservable vacuum energy $E(0)$ has been subtracted from the hamiltonian. Before and after the collision, i.e. for $t \rightarrow \pm \infty$, the state (4.15) must be describable as corresponding to the simultaneous presence of various particles widely separated from each other. These particles, 
however, must be physical particles moving in the physical vacuum, i.e. entities to be defined in terms of the interaction and not of the free hamiltonian alone, as would be the case in ordinary collision theory. The form (4.7) of the one particle states suggests a natural description for such a timedependent situation where $n$ particles are widely separated from each other and move independently. It is naturally represented by a wave function

$$
\left.\psi(t)=\int 0_{k_{1}} \ldots 0_{k_{n}} \mid 0\right) \mathrm{d} k_{1} \ldots \mathrm{d} k_{n} \exp \left[-i\left(E_{k_{1}}+\ldots E_{k_{n}}\right) t\right] c^{\prime}\left(k_{1} \ldots k_{n}\right)
$$

where the coefficients $c^{\prime}\left(k_{1} \ldots k_{n}\right)$ are chosen to give for each particle its own one-particle wave packet in exactly the same way as would be done for free particles. In (4.17) the $0_{k}$ commute with each other. A wave function as (4.17) can of course not be a solution of the Schrödinger equation (4.16), but it should verify it at all times for which the $n$ physical particles are far from each other, i.e. essentially for large $|t|$, or for $t$ finite and $c^{\prime}\left(k_{1} \ldots k_{n}\right)$ with rapidly varying phases. That this is indeed the case follows from the results of I, section 5 . The states $0_{k_{1}} \ldots 0_{k_{n}} \mid 0$ ) are, for our field-theoretical system, the asymptotically stationary states there defined, because the diagrams contributing to the diagonal part of the resolvent are composed exclusively of vacuum and one-particle components (see also III, section 13).

The determination of the incoming and outgoing particles in a collision process described by (4.15) is now reduced to a proof that $\varphi_{ \pm}(t)$ approaches the form (4.17) for $t \rightarrow \pm \infty$ and to the calculation of the corresponding coefficients $c^{\prime}\left(k_{1} \ldots k_{n}\right)$. This has been accomplished in II, section 3. Convergence in Hilbert space being taken in the usual sense of the norm approaching zero, one has under the assumption that the amplitude $c\left(k_{1} \ldots k_{n}\right)$ is a smooth function of its arguments

$$
\begin{aligned}
& \lim _{t \rightarrow-\infty}\left[\varphi_{++}(t)-\right. \\
& \left.\quad-\int 0_{k_{1}} \ldots O_{k_{n}} \mid 0\right) \mathrm{d} k_{1} \ldots \mathrm{d} k_{n} \exp \left[-i\left(E_{k_{1}}+\ldots E_{k_{n}} t\right] c\left(k_{1} \ldots k_{n}\right)\right]=0, \\
& \lim _{t \rightarrow+\infty}\left[\varphi_{+}(t)-\sum_{n^{\prime}>1} \int 0_{k_{1}^{\prime}} \ldots O_{k^{\prime}{ }^{\prime}} \mid 0\right) \mathrm{d} k_{1}^{\prime} \ldots \mathrm{d} k_{n^{\prime}}^{\prime} . \\
& \left.\quad \exp \left[-i\left(E_{k^{\prime}}+\ldots E_{k_{n^{\prime}}}\right) t\right] .\left\langle k_{1}^{\prime} \ldots k_{n^{\prime}}^{\prime}|S| k_{1} \ldots k_{n}\right\rangle c\left(k_{1} \ldots k_{n}\right) \mathrm{d} k_{1} \ldots \mathrm{d} k_{n}\right]=0,
\end{aligned}
$$

with the following expression for the $S$-matrix

$$
\begin{aligned}
& \left\langle k_{1}^{\prime} \ldots k_{n^{\prime}}^{\prime}|S| k_{1} \ldots k_{n}\right\rangle=\delta_{n n^{\prime}} \delta\left(k_{1}^{\prime}-k_{1}\right) \ldots \delta\left(k_{n}^{\prime}-k_{n}\right) \\
& \quad-2 \pi i \delta\left(E_{k k_{1}}+\ldots E_{k_{n^{\prime}}^{\prime}}-E_{k_{1}}-\ldots E_{k_{n}}\right)\left(N_{k^{\prime}} \ldots N_{k_{n^{\prime}}{ }^{\prime}} N_{k_{1}} \ldots N_{k_{n}}\right)^{t} . \\
& . \sum_{\delta}\left\langle k_{1}^{\prime} \ldots k_{n^{\prime}}^{\prime}\left|\left\{V-V R_{E+i 0} V\right\}_{\delta}\right| k_{1} \ldots k_{n}\right\rangle .
\end{aligned}
$$

$E$ stands for $E_{k_{1}}+\ldots E_{k_{n}}$ and the sum extends over all diagrams $\delta$ with the following properties: $\delta$ has no vacuum components; and neither $\delta$ nor any of the smaller diagrams obtained by erasing in $\delta$ all what is on the left or on the right of an intermediate state, are entirely composed of one- 
particle components. (4.20) follows from elimination of vacuum components in equation (6.5) of $I$, using (3.3). One has also

$$
\begin{array}{r}
\Sigma_{\delta}\left\langle k_{1}^{\prime} \ldots k_{n^{\prime}}^{\prime}\left|\left\{V-V R_{E+i 0} V\right\}_{\delta}\right| k_{1} \ldots k_{n}\right\rangle=\left(N_{k^{\prime}} \ldots N_{k_{n}^{\prime}} N_{k_{1}} \ldots N_{k_{n}}\right)^{-1} . \\
. \lim _{l \rightarrow E}(l-E)^{2} \Sigma_{\gamma}\left\langle k_{1}^{\prime} \ldots k_{n^{\prime}}^{\prime}\left|\left\{R_{l}\right\}_{\gamma}\right| k_{1} \ldots k_{n}\right\rangle
\end{array}
$$

under the conditions

$$
\operatorname{Im}(l)>0, E_{k_{1}}+\ldots E_{k_{n}}=E_{k_{1}^{\prime}}+\ldots E_{k^{\prime} n^{\prime}}=E
$$

and where $\gamma$ represents all diagrams without vacuum components, not entirely composed of one-particle components. Equations similar to (4.18) and.(4.19) hold for $\varphi_{-}(t)$ : the limits $t \rightarrow \pm \infty$ must be interchanged and the $S$-matrix must be replaced by its inverse, which is at the same time its hermitian conjugate.

None of the equations (4.5), (4.8), (4.11) and (4.20) or their alternatives are in the most convenient form for actual calculations. Just as for the Feynman-Dyson diagrams of the time-dependent formalism, many reductions can be carried out. They all follow from simple applications of (3.3) but will not be described here.

5. A remark on renormalization constants. To carry out renormalization in the time-independent formalism presented above, one would proceed as follows. One would eliminate all unperturbed energies by expressing them in terms of the observable energies $E_{k}$, thus performing mass renormalization. One would eliminate the original coupling constant $g$ by expressing it in terms of an observable coupling constant $g^{\prime}$ to be defined as a measure of the strength of one special collision process, low energy scattering for instance. In the most favorable case, examplified by electrodynamics, one would obtain in this way, for all elements of the $S$-matrix, perturbation expansions with coefficients which remain finite in the limit of an infinite cut-off. Other expressions like the expansions of physical states in bare states, as given in section 4, would of course not have this property. The renormalization procedure can be carried out in principle for a non-covariant theory as well as for a covariant one, although of course in the latter case the use of covariance considerations simplifies the calculations to a very large extent.

When following the above procedure one would find for the renormalized coupling constant $g^{\prime}$ an expression involving $g$, some of the constants $N_{k}$ introduced in section 3 and a vertex constant $N^{\prime}$ which we shall not define explicitly here. In electrodynamics, the constant $N^{\prime}$ cancels out exactly with the constant $N_{k}$ for the electron (this follows from an identity analogous to the Ward identity ${ }^{5}$ ) of the covariant formalism) and one finds for the renormalized charge

$$
e^{\prime}=e N_{0}^{\frac{1}{2}}
$$


where $e$ is the unrenormalized charge and $N_{0}$ the constant $N_{k}$ for a photon of vanishing momentum.

As first shown by Dyson ${ }^{2}$ ), the covariant formalism based on the time integration of the equation of motion leads to a relation between renormalized and unrenormalized coupling constants which involves certain constants usually denoted by $Z$ (sometimes called the wave function renormalization constants) as well as a properly defined vertex constant (different from the $N^{\prime}$ mentioned above). In electrodynamics, according to Ward's identity ${ }^{5}$ ), the vertex constant cancels with the $Z$ constant of the electron (usually denoted by $Z_{2}$ ) and one finds

$$
e^{\prime}=e Z_{3}^{\frac{1}{2}}
$$

where $Z_{\mathbf{3}}$ is the $Z$-constant for the photon.

Although we shall show that for a photon of vanishing momentum $N_{0}=Z_{3}$, so that (5.1) and (5.2) are equivalent equations, the constants $Z$ of the covariant formalism are in general different from our constants $N_{k}$. We want to clarify this difference in the present section. For the neutral scalar bosons studied in sections 2 to 4 one obtains the constant $Z$ by considering the complete Feynman propagation function $\Delta_{F}^{\prime}\left(x-x^{\prime}\right)$ of a particle between two points $x, x^{\prime}$ of space time. This function is defined as the total contribution, in the sense of Dyson's theory, of all connected Feynman diagrams with one line ending at $x$, one at $x^{\prime}$, and without further external lines. It has the form

$$
\Delta_{F}^{\prime}(x)=Z\left[\Delta_{F}\left(x, \mu^{\prime}\right)+\int_{\kappa_{0}}^{\infty} \rho(\kappa) \mathrm{d} \kappa \Delta_{F}(x, \kappa)\right] .
$$

In this formula, which defines $Z, \mu^{\prime}$ is the renormalized mass, $\kappa_{0}$ a mass value larger than $\mu^{\prime}$ and $\rho(\kappa)$ a positive function defined for $\kappa>\kappa_{0} . \Delta_{F}(x, \kappa)$ denotes the Feynman propagator for free bosons of mass $\kappa$ :

$$
\Delta_{F}(x, \kappa)=\frac{i}{16 \pi^{4}} \int \frac{\exp \left[i\left(k_{1} x_{1}+k_{2} x_{2}+k_{3} x_{3}-k_{0} x_{0}\right)\right]}{k_{1}^{2}+k_{2}^{2}+k_{3}^{2}-k_{0}^{2}+\kappa^{2}-i 0} \mathrm{~d} k_{0} \mathrm{~d} k_{1} \mathrm{~d} k_{2} \mathrm{~d} k_{3} .
$$

The above definition of $Z$ implies $0<Z<1^{*}$ ). From (5.3) one finds for $x_{0}$ positive and large

$$
\int \mathrm{d} \boldsymbol{x} \exp (i k, \boldsymbol{x}) \Delta_{F}^{\prime}(x) \simeq Z \exp \left(-i E_{k} x_{0}\right) / 2 E_{k}
$$

with $E_{k}=\left(|k|^{2}+\mu^{\prime 2}\right)^{1}$. We have written $\boldsymbol{x}$ for the space part of $x$ and $k \cdot \boldsymbol{x}$ for its scalar product with the three-momentum $k$.

The definition of $\Delta_{F}^{\prime}$ in terms of Feynman diagrams can be directly written out in our non-covariant notation. For $x_{0}>x_{0}^{\prime}$ one has

$$
A_{F}^{\prime}\left(x-x^{\prime}\right)=\sum_{\delta}\left\langle 0\left|\left\{U_{\infty} A_{s}(\boldsymbol{x}) U_{x_{0}-x_{0}^{\prime}} A_{s}\left(\boldsymbol{x}^{\prime}\right) U_{\infty}\right\}_{\delta}\right| 0\right\rangle,
$$

$\left.{ }^{*}\right)$ See ref. 6, where another, equivalent, definition of $\Delta_{F}{ }_{F}$ is used: $\Delta_{F}^{\prime}\left(x-x^{\prime}\right)$ is the expectation value of the time ordered product $P\left[A_{H}(x) A_{H}\left(x^{\prime}\right)\right]$ for the physical vacuum, $A_{H}$ denoting the Heisenberg field operator. 
where for each $U_{\infty}$ the infinite time limit must be taken in the sense of the mean. $A_{s}(\boldsymbol{x})$ is the Schrödinger (time-independent) field operator in the point $\boldsymbol{x}$ of space. $U_{t}$ is the operator of motion (2.11), to be inserted in (5.5) as the well known series expansion in the interaction

$$
\begin{aligned}
U_{t}= & U_{t}^{0}-i \int_{0}^{t} \mathrm{~d} t_{1} U_{t-t_{1}}^{0} V U_{t_{1}}^{0}+\ldots \\
& +(-i)^{n} \int_{0}^{t} \mathrm{~d} t_{n} \int_{0}^{t_{n}} \mathrm{~d} t_{n-1} \ldots \int_{0}^{t_{2}} \mathrm{~d} t_{1} U_{t-t_{n}}^{0} V U_{t_{n}-t_{n-1}}^{0} \ldots V U_{t_{1}}^{0}+\ldots
\end{aligned}
$$

where we have put

$$
U_{t}^{0}=\exp \left(-i H_{0} t\right)
$$

The sum in (5.5) extends over the contributions of diagrams $\delta$ of the same general type as the diagrams introduced in section 3, except for the fact that in addition to the vertices there considered two new vertices occur, corresponding to the operators $A$. They are vertices with one line only ending in them. Among the diagrams of this type, the summation in (5.5) is specifically extended over all connected diagrams without external lines.

Consider now instead of (5.5) the following function

$$
C\left(x-x^{\prime}\right)=\Sigma_{\delta}\left\langle 0\left|\left\{A_{s}(\boldsymbol{x}) U_{x_{0}-x_{0}^{\prime}} A_{s}\left(\boldsymbol{x}^{\prime}\right)\right\}_{\delta}\right| 0\right\rangle,
$$

the sum extending over the same diagrams as in (5.5). In contrast to (5.5), however, the only diagrams contributing to (5.8) are those with the two special vertices belonging to the $A_{s}$ located at the extreme right and left. Erasing these special vertices one would obtain the one-particle components considered in section 3. One therefore expects a simple relation to exist between $C\left(x-x^{\prime}\right)$ and our function $C_{l}(k)$ of section 3 . Remembering that

$$
A_{s}(\boldsymbol{x})=\Sigma_{k}\left(2 \Omega \varepsilon_{k}\right)^{-\frac{1}{2}} q_{k} \exp (i k \cdot \boldsymbol{x}) \text { with } q_{k}=a_{k}+a_{-k}^{*}
$$

and rewriting the definition of $C_{l}(k)$ as

$$
C_{l}(k)=\Sigma_{\delta}\left\langle 0\left|\left\{q_{k} R_{l} q_{-k}\right\}_{\delta}\right| 0\right\rangle
$$

with the same diagrams $\delta$ as in (5.8), one easily finds

$$
\int \mathrm{d} \boldsymbol{x} \exp (i k . x) C(x)=\left(i / 4 \pi \varepsilon_{k}\right) \int \mathrm{d} l \exp \left(-i l x_{0}\right) C_{l}(k) .
$$

The contour integration over $l$ encircles counterclockwise a sufficiently large portion of the real axis. (5.11) gives in particular for $x_{0} \rightarrow \infty$

$$
\int \mathrm{d} \boldsymbol{x} \exp (i k \cdot \boldsymbol{x}) C(x) \simeq N_{k} \exp \left(-i E_{k} x_{0}\right) / 2 \varepsilon_{k} .
$$

Comparison of (5.8), (5.12) with (5.5), (5.4) indicates the analogies and differences between the usual propagator and our function $C_{l}(k)$, and between the constants $Z$ and $N_{k}$ defined in terms of them. When the interaction is such that it does not affect the vacuum, the expressions (5.5) and (5.8) coincide, so that $Z$ and $N_{k}$ become identical. This circumstance, which never 
occurs in a relativistic local theory, is very common in simplified non relativistic theories as the Lee model and its extensions ${ }^{7}$ ), and the Chew model for the nucleon-pion system ${ }^{8}$ ). In all cases, however, where the physical vacuum $(0)$ differs from the bare vacuum, the functions $\Delta_{F}^{\prime}(x)$ and $C(x)$, and generally also the constants $Z$ and $N_{k}$, are different. As an example we quote the second order values of $Z$ and $N_{k}$ for an electron of momentum zero in interaction with the Maxwell field:

$$
\begin{gathered}
Z_{2}=1-(e / 4 \pi)^{2}\left[(2 \pi)^{-1} \int\left(m^{2}+|k|^{2}\right)^{-3 / 2} \mathrm{~d} k-4 \int_{0}^{1} x^{-1} \mathrm{~d} x+\frac{11}{2}\right], \\
\left(N_{0}\right)_{e l}=1+(e / 4 \pi)^{2} m \pi^{-1} \int|k|^{-3}\left[\left(|k|^{2}+m^{2}\right)^{\frac{1}{2}}+|k|\right]^{-1} \mathrm{~d} k .
\end{gathered}
$$

$k$ is a three dimensional momentum and $\mathrm{d} k$ the corresponding volume element. Both expressions have the same logarithmic infra-red divergence. Only the first one diverges at high momenta.

There is nonetheless one type of particle for which the constants $Z$-and $N_{k}$ are always simply related: for a particle with bare and renormalized masses both zero, the constant $Z$ is equal to the value $N_{0}$ of $N_{k}$ at $k=0$. As announced earlier, this happens for the photon and entails the identity of (5.1) and (5.2). The proof of our assertion runs as follows. Consider the quantity

$$
\left\langle 0\left|\left\{q_{k} U_{t+t^{\prime}+l^{\prime \prime}} q_{-k}\right\}_{\delta}\right| 0\right\rangle
$$

for a connected diagram $\delta$ without external lines, the special vertices belonging to the $q$ 's being located at the extreme right and left. If the unperturbed energy $\varepsilon_{k}=|k|$ is very small, the value of (5.13) does not change appreciably when we shift $q_{k}$ and $q_{-k}$ to other places in between the operators $V$ occurring in the expansion of $U$ and modify accordingly the diagram $\delta$ by shifting the special vertices. On the basis of the identity

$$
U_{t+t^{\prime}+t^{\prime \prime}}=U_{t^{\prime \prime}} U_{t^{\prime}} U_{t}
$$

one can establish by such shifting that, for $|k|$ very small,

$$
\Sigma_{\delta}\left\langle 0\left|\left\{q_{k} U_{t+t^{\prime}+t^{\prime \prime}} q_{-k}\right\}_{\delta}\right| 0\right\rangle=\Sigma_{\delta}\left\langle 0\left|\left\{U_{t^{\prime \prime}} q_{k} U_{t^{\prime}} q_{-k} U_{t}\right\}_{\delta}\right| 0\right\rangle
$$

both sums extending over all connected diagrams without external lines. There is a time $t_{0}$ independent of $k$ (in electrodynamics it is of the order of the reciprocal electron mass) such that for $t \gg t_{0}, t^{\prime \prime} \gg t_{0}$ one can replace $U_{t}$ and $U_{t^{\prime \prime}}$ by $U_{\infty}$ in the righthand side of (5.14). If in addition $t^{\prime} \gg t_{0}$, this expression reduces to (5.4) with $x_{0}$ replaced by $t^{\prime}$. On the other hand, for $t+t^{\prime}+t^{\prime \prime} \gg t_{0}$, the lefthand side of (5.14) reduces to (5.12) with $x_{0}$ replaced by $t+t^{\prime}+t^{\prime \prime}$. We thus get

$$
N_{k} \exp \left[-i E_{k}\left(t+t^{\prime}+t^{\prime \prime}\right)\right]=Z \exp \left(-i E_{k} t^{\prime}\right)\left(\varepsilon_{k} / E_{k}\right) .
$$

under the assumptions $\varepsilon_{k}=E_{k}=|k| \ll\left(t+t^{\prime}+t^{\prime \prime}\right)^{-1}, t, t^{\prime}$ and $t^{\prime \prime} \gg t_{0}$. Both exponentials are approximately equal to one, and we get the an- 
nounced identity $N_{0}=Z$. This conclusion applies to the photon: the modifications required by the vector character of this particle do not affect the above derivation.

We end this section with two remarks. One concerns equation (5.1), or equivalently the result just established for the photon. It gives an interesting interpretation for the ratio $\left(e^{\prime} \mid e\right)^{2}$ of the renormalized and unrenormalized values of the fine structure constant. This ratio is the photon constant $N_{0}$, which we have shown (section 4) to be the probability to find the bare photon state in the physical photon state of vanishing momentum, divided by the probability of finding the bare vacuum in the physical vacuum. No such simple probabilistic interpretation could be given to the constant $Z_{3}$ on the basis of its definition in the usual formalism. Our second remark concerns a methodological question: could one derive the stationary states of interacting fields by means of the time dependent perturbation formalism conventionally used for the $S$-matrix? This could indeed be done by integrating the equation of motion for the time interval $-\infty<t<0$ using an adiabatic switching on of the interaction. In this process, each unperturbed stationary state $|\alpha\rangle$ is transformed into the corresponding perturbed stationary state $|\alpha\rangle_{+}$. The time dependent procedure just mentioned which has already been used for the vacuum state by Gell-Mann. and Low ${ }^{9}$ ) would give, less directly, results equivalent to ours, the difference amounting essentially to a Fourier transformation. One should note that, just as our method, the time dependent procedure is not covariant: in contrast with the $S$-matrix calculation which integrates over the time interval $-\infty<t<+\infty$, it involves an hypersurface $t=0$ of space time. For the same reason the determination of the stationary states cannot be carried out in terms of the propagators $\Delta_{F}^{\prime}$ which are so well suited for the $S$-matrix. To our knowledge the problem of finding a fully covariant perturbation expression for the stationary states of relativistic interacting fields has not been investigated.

Received 9-12-57

\section{REFERENCES}

1) Van Hove, L., Physica 21 (1955) 901 and 22 (1956) 343;

Hugenholtz, N. M., Physica 23 (1957) 481, quoted in the text as I, II, III respectively.

2) Dyson, F. J., Phys. Rev. 75 (1949) 486 and 1736.

3) Van Hove, L., Physica 23 (1957) 441.

4) Wick, G. C., Rev. mod. Phys. 27 (1955) 339.

5) Ward, J. C., Phys. Rev. 78 (1950) 182.

6) Lehmann, H., Nuovo Cimento 11 (1954) 342.

7) Lee, T. D., Phys. Rev. 95 (1954) 1329 ; Kallén, G. and Pauli, W., Dan. mat. fys. Medd. 30 (1955) no. 7; Ruijgrok, Th. W. and Van Hove, L., Physica 22 (1956) 880.

8) Chew, G. F., Phys. Rev. 94 (1954) 1748 and 1755; ibidem 95 (1954) 1669; see also Wick, ref. 4.

9) Gell-Mann, M. and Low, F., Phys. Rev. 84 (1951) 350 (appendix). 